\title{
The Sonic Surface in the Inter-Blade Channel of the Last Stage Rotor Wheel in the Steam Turbine of Large Output
}

\author{
Martin Luxa ${ }^{1, *}$ \\ ${ }^{1}$ Institute of Thermomechanics of the Czech Academy of Sciences, Dolejškova 5, 18200 Prague, \\ The Czech Republic
}

\begin{abstract}
The paper deals with sonic surface in a modern turbine wheel consisting of non-prismatic ultra long blades. The whole inter-blade channel is choked. Different positions and shapes of the sonic line in particular cross-sections along the span are observed. The sensitivity of sonic line formation to small changes of effective shape of the inter-blade channel in the root section and the influence of inlet angle, stagger angle and pitch/chord ratio in the tip section are discussed. The problematic of sonic line development in the case of supersonic inlet flow filed is also described. The presented work is based on results of theoretical, experimental and numerical approaches.
\end{abstract}

\section{Introduction}

The modern rotor blade wheels in the last stages of low-pressure cylinders, that are embodied in the steam turbines of large energy output, usually consist of ultra long blades. The thermodynamic conditions in the last stage of the low-pressure cylinder, sizeable diameter of the cylinder, finally the common nominal rotational frequency $\left(50 . s^{-1}\right)$ of the wheel, result in transonic flow field in the inter-blade channel and outlet part of the blades assembly. Under specific conditions, the transonic (or even supersonic) flow field appears at the blade wheel inlet in the tip region. This description is well illustrated in the Figure 1, showing an example of numerical simulation of the flow field in the last stage consisting of ultra long turbine titanium blades $1375 \mathrm{~mm}$.

The knowledge of sonic surface shape (or sonic line - regarding the particular section in the $3 \mathrm{D}$ inter-blade channel) is important. It usually represents the boundary condition for the supersonic flow field solution and is the boundary line of the region in the transonic flow, wherein the solution of hyperbolic partial differential equations is supposed. The shape of sonic line and thus the entire development of supersonic flow is sharply dependent on the geometrical parameters of the cascade, in particular on the pitch to chord ratio, on the angle of flow turning etc. In the Prandtl- Meyer problem (simple supersonic expansion), the sonic line is always orthogonal to the stream lines upstream. It means that

\footnotetext{
*Corresponding author: luxa@it.cas.cz
} 
the stream line curvature is zero in the sonic point. This assumption is often used in such a way, that the sonic line is identical with the geometric throat of the considered channel.

This condition is fulfilled rarely in real flows. The solution of the shape of sonic line in a channel is proposed e.g. by Safarik [2], [3], Shapiro [4] or Thompson [5]. Interesting overview of various analytical solutions of sonic line shape in symmetric and asymmetric channels provides the work of Dvorak [6]. The solution of the shape of sonic line in the narrowest part of an inter-blade channel is based on knowledge of the channel curvature in the region of channel throat. The concept of a steady flow of ideal inviscid compressible fluid is applied, which is described by linearised potential equation. These analytical solutions of sonic line usually approximate the curvature of channel surfaces defining the aerodynamic throat by circular curves.

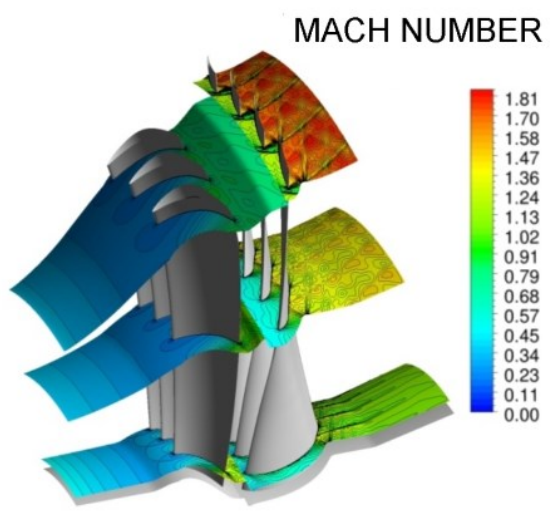

Fig. 1. Result of numerical simulation of flow in the last stage of the low pressure cylinder in the steam turbine Škoda consisting of ultra long titanium rotor blades $1375 \mathrm{~mm}$ - visualisation of the Mach number distribution [1].

The data regarding the shape and position of sonic line are often adopted from numerical simulation or from experiment. Very useful experimental method for the sonic line detection is interferometric method (in 2D flow fields modelling). Advantageous seems application of so called infinite interferometric fringe method [4], where the fringes in interferometric chart are directly related to regions of constant refractive index. The mutual relation of refractive index and density of the fluid is given by Lorenz -Lorentz law. This non-linear law can be replaced by simpler linear Gladstone - Dale law if the flowing fluid is dry air. Thus, we can match the appropriate density with each fringe in the interferometric picture and this procedure makes it naturally also possible to determine the position and shape of sonic fringe in the flow field. It is necessary to bear the isoentropic condition in mind, i.e. the evaluation of interferometric fringes is usually related to the total pressure at inlet of the investigated inter-blade channel. Distortion of resulting interferometric picture is especially important in boundary layers and shear layers.

\section{Sonic lines in selected sections of an inter-blade channel}

The sonic line joins the surfaces of adjacent blades under nominal conditions of the flow in all sections along the inter-blade channel in rotor row of the last stage. The exception can represent the tip region of ultra long rotor blades with supersonic flow at the inlet. The sonic line closes around the leading edge in this case (started supersonic flow), near the stagnation point. The situation between the subsonic and started supersonic inlet flow (unstarted supersonic flow) represents much more complex issue [6] with unsteady 
behaviour and manifestation of flow field instability. The next paragraphs are focused mainly on flow fields in such turbine cascade geometries, where the inlet flow is subsonic, and thus the sonic line connects the pressure and suction side of the adjacent profiles.

\subsection{Root section}

A considerable sensitivity of sonic line shape on the inter-blade channel shaping is typicall for geometries representing root sections of rotor rows of the last stage. The inlet Mach number in nominal regimes is usually relatively very low. Its value commonly lies under $M_{l}=0.4$. The convergent part of the inter-blade channel is formed so that downstream the inlet part, relatively very long part of inter-blade channel with significantly small narrowing is situated. This convergent part ends in the region of geometrical throat and the following divergent part of the inter-blade channel is usually very short. The supersonic expansions take place mainly in the exit part of the profile cascade. Considering small changes of inlet/throat cross section ratio along the central line of practically convergent inter-blade channel (Figure 2), the small changes of profile shape in region of aerodynamic throat together with different parameters of boundary layers have considerable impact not only on the shape of the sonic line but also on the supersonic expansion downstream.

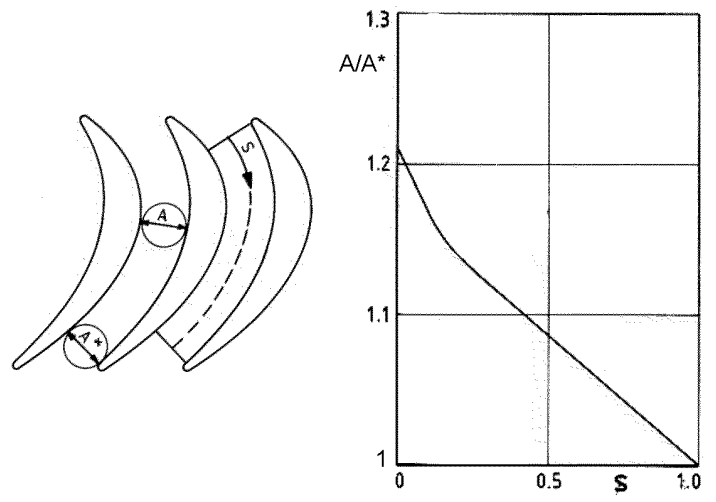

Fig. 2. Example of root section designed in VKI with depicted dependence of inter-blade channel contraction on the position along the channel central line [7].

An example of experimental modelling of flow fields in two variants of root section supplemented by data from numerical simulation is shown in the Figures 3 and 4. Both variants were intended for the same diameter of rotor wheel and the contours of profiles were similar; however, the number of blades in the wheels was different. The small difference in the number of blades generates very small changes in the pitch/chord ratio parameter (the second decimal place). The interferograms of both variants are in the Figure 3 with highlighted sonic line. The experimental data regarding the measurement of the variant with smaller pitch/chord ratio $(s / c=0.2603)$ corresponds well with numerical simulations in the Figure 4. However, the sonic line in the numerical simulation data of flow with larger pitch/chord ratio $(s / c=0.2732)$ is bended in a different way in comparison with experimental data. The reasons of this difference can reside in an application of chosen calculation method - the flow field was considered fully turbulent. The turbulent model $k \omega-$ $S S T$ with non-reflective boundary condition in commercial software was applied. The more detailed numerical modelling of the boundary layer on the suction side of the profile with application of transition models of turbulence might bring more accurate results. This research was performed for nominal inlet flow angle where the value of incidence angle was positive $\left(\iota=+14^{\circ}\right)$. This positive incidence is linked to the application of the 
profile on straight fir-tree dovetail. The positive incidence leads to small decreasing of static pressure along the suction side of the profile in a restricted region downstream the stagnation point. This expansion is downstream followed by large region of practically constant static pressure. This results not only in complicated processes in the boundary layer on the suction side, but also in important changes of effective shape of the inter-blade channel.

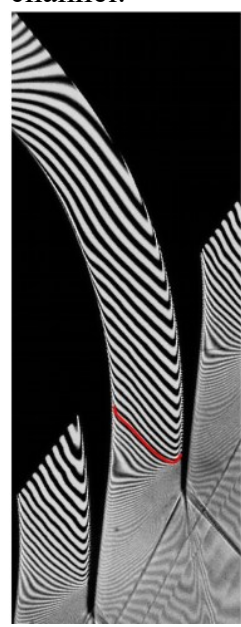

a) $s / c=0.2603$

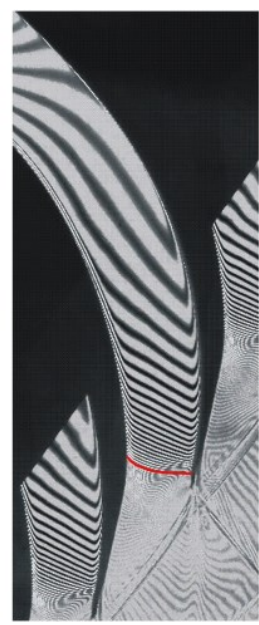

b) $s / c=0.2732$

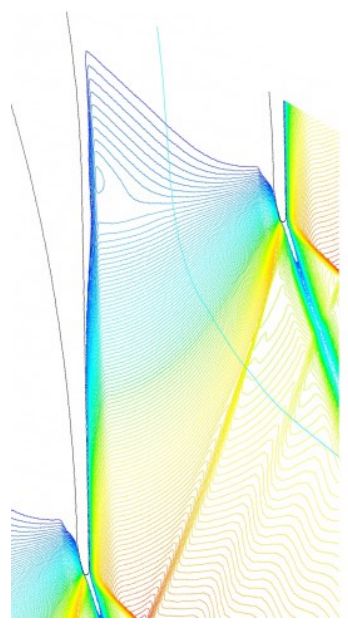

a) $s / c=0.2603$

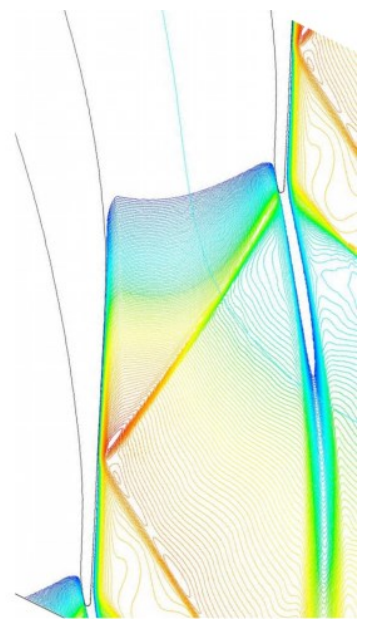

b) $s / c=0.2732$

Fig. 3. Impact of small changes of pitch/chord ratio on the shape of sonic line in the root section, interferometric measurements, exit Mach number

Fig. 4. Impact of small changes of pitch/chord ratio on the shape of sonic line in the root section, numerical simulation, exit Mach number $M_{2 i s} \sim 1.6$, isolines of isoentropic Mach number [9]. $M_{2 i s} \sim 1.8[8]$.

The next example shows other variant of this root section. The pitch/chord ratio is the same as in the variant $b(s / c=0.2732)$, the shape of new profile is slightly more arched in the suction side. The arrangement of new profiles in the cascade is different, i.e. stagger angle is set in such a way, that the incidence is zero in the contrast to the original variant.

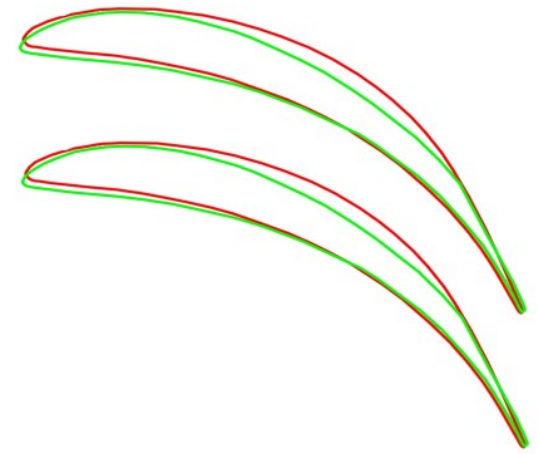

Fig. 5. Comparison of original (green line) and improved (red line) variant of the root profile.

Both profiles are compared in the Figure 5, the cascades in the figure are mutually turned. The interferometric picture together with result of numerical simulation is compared in the Figure 6 . The sonic line is highlighted by red line. The calculation was carried out with commercial software with application of turbulent model $k \omega-S S T$ with non-reflective boundary condition (the same procedure as in the foregoing example). One can observe the 
agreement of the position of sonic point on the suction side in both research approaches. This good agreement between numerical study and experimental data is certainly caused by more favourable static pressure distribution along the suction side in the subsonic part of the inter-blade channel - the region of static pressure stagnation is eliminated. Substantial is also the neutral incidence angle for boundary layer development on the suction side.

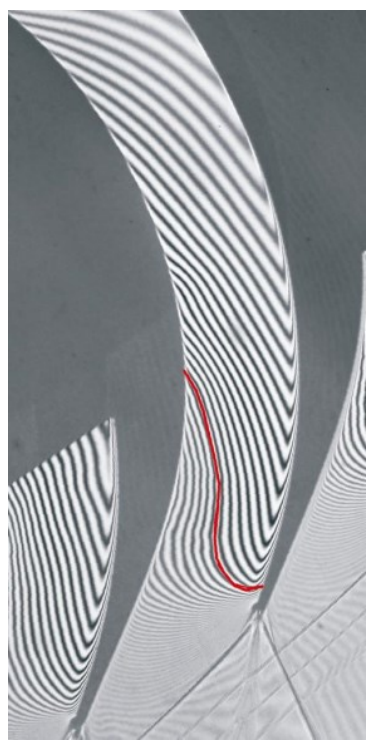

Fig. 6. The shape and position of sonic line in improved version of root section $(s / c=0.2732)$. Interferometric measurement, exit Mach number $M_{2 i s} \sim 1.7$, incidence angle $\imath=0^{\circ}[10]$.

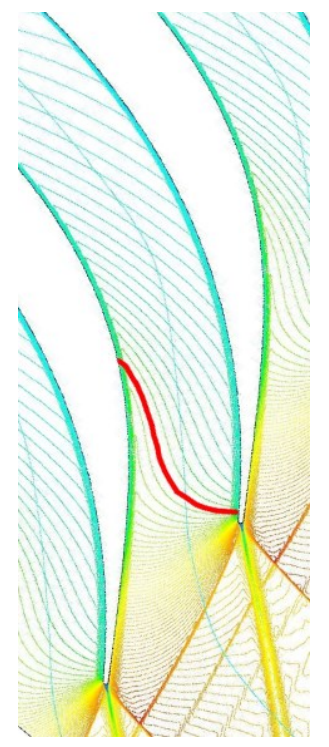

Fig. 7. The shape and position of sonic line in improved version of root section $(s / c=0.2732)$. CFD calculation, exit Mach number $M_{2 i s} \sim 1.7$, incidence angle $t=0^{\circ}[11]$.

These examples of sonic line in the root section well illustrate the extraordinary sensitivity of the sonic lane shaping in the inter-blade channels not only on profile geometry but also on small changes of effective channel contours, which are related mainly to boundary layer development. These root channels are generally characterized as relatively narrow, relatively long and mildly convergent (or convergent-divergent) channels. The experience with application of numerical methods shows the necessity to use proper models of turbulence (including transition models). The CFD simulations, which consider fully turbulent flow field, can lead to inaccurate results in terms of the sonic line and pressure distribution along the profile.

\subsection{Middle section}

Along the inter-blade channel in the radial direction, the pitch/chord ratio increases and contrarily, the profile chord usually somewhat decreases. These two facts together with changes of stagger angle (the long rotor blade are non-prismatic) lead to increasing of interblade channel width and concurrently to considerable reduction of its length. The aerodynamic throat moves upstream to the middle area of the profile cascade (see Figure 8). The maximum of the curvature of the camber line moves in the leading edge direction. The inlet Mach number increases along the blade height. The real values of the inlet Mach number ranges still in subsonic region. The example of common shape of sonic line in the mid-section is shown in the interferometric picture in the Figure 9. A typical 
distribution of isentropic Mach number near the profile in profile cascade representing the mid-section is depicted in the Figure 10.

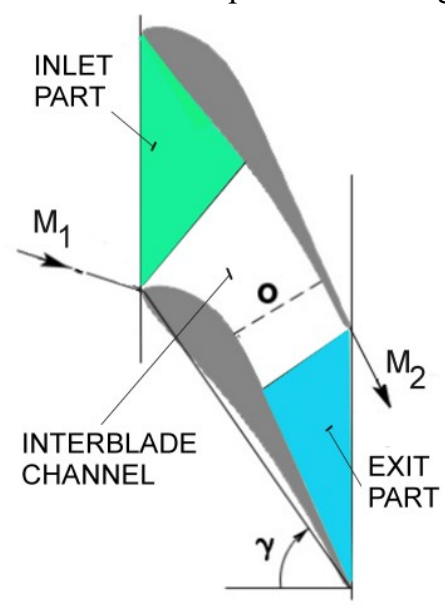

Fig. 8. Geometric configuration of typical middle section in the rotor wheel consisting of long blades.

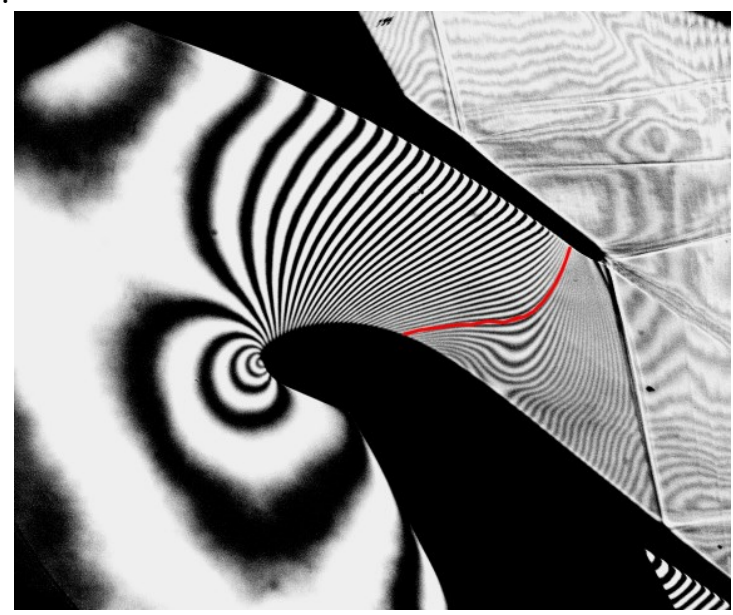

Fig. 9. The sonic line in the inter-blade channel of the middle section. Interferometric measurement, exit Mach number $M_{2 i s} \sim 1.35$, incidence angle $\imath=0^{\circ}$.

Approximately in the middle of blade height the special damping device tie-boss is often situated. The influence of this body on the sonic line in the adjoining sections along the inter-blade channel is described in [12].

\subsubsection{Recompression}

The shaping of the profiles intended for application in the middle of the long rotor blade is often a reason for progressive development of recompression phenomenon in the supersonic part of the flow field next to the sonic line.

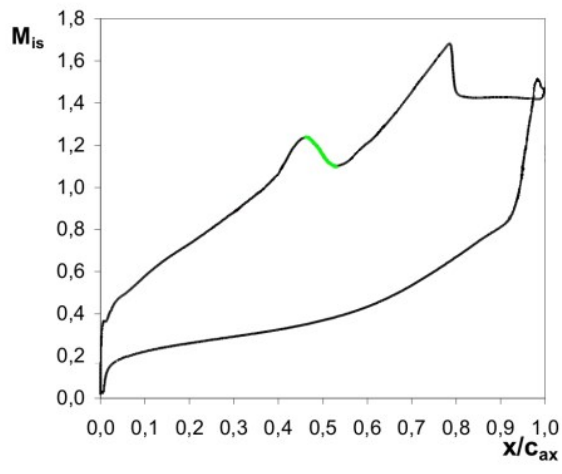

Fig. 10. The isentropic Mach number distribution along the blade of the middle section. Numerical simulation, exit Mach number $M_{2 i s} \sim 1.323$, incidence angle $\imath=0^{\circ}$, the recompression highlighted in green [13].

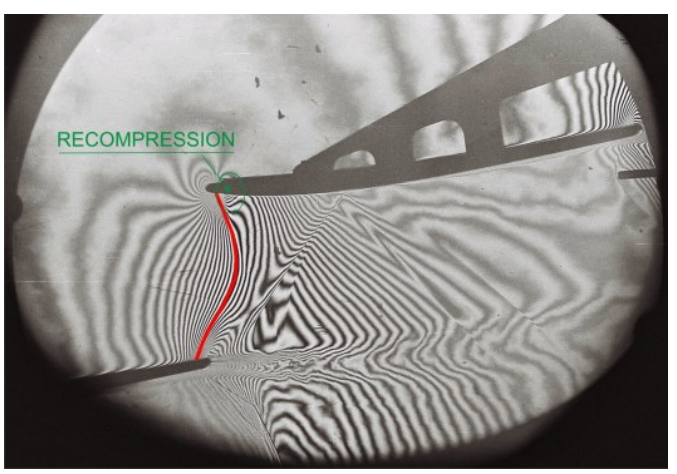

Fig. 11. The sonic line and recompression field in a tip section of the rotor wheel consisting of long blades. Interferometric measurement, exit isentropic Mach number $M_{2 i s} \sim 1.28$, inlet Mach number $M_{1} \sim 0.790$ [14].

The recompression area often appears in supersonic flow field in different sections along the ultra long blade, even in the tip sections - (if the tip profile has usually bended 
central line and the inlet Mach number is subsonic). The supersonic compression under transonic expansion (so called recompression) is a characteristic consequence of application of line segment in the shape of the suction side. This segment is attached up the upstream previous convex curve and is placed usually near the geometrical throat of the profile cascade. Although the sudden change of the shape curvature lies in the supersonic part of the flow field downstream the sonic line, the suitable geometrical configuration can guarantee the smooth increasing of static pressure, which is followed by ongoing supersonic expansion. The dissipative shock wave does not appear. On the other hand, the local increasing of the static pressure causes moderate decreasing of pressure force on the profile. The recompression appears also in other sections of wheels with long blades (example of the tip section with recompression area is shown in the Figure 11).

The sudden change of the suction side curvature just downstream the sonic line leads to the origin of reflected compression waves. The expansion waves, which originate on the convex part of the suction side, reflect from the sonic line as the compression waves.

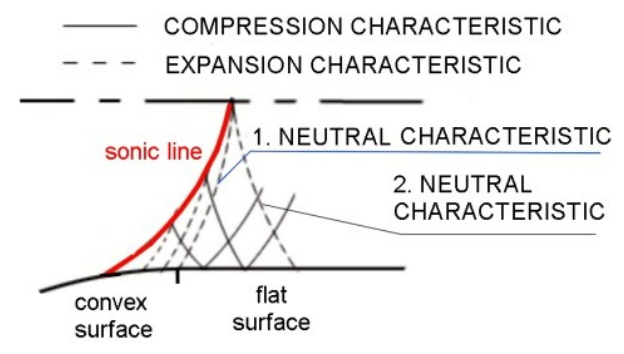

Fig. 12. Principle of the compression field formation in the supersonic flow downstream the sonic line.

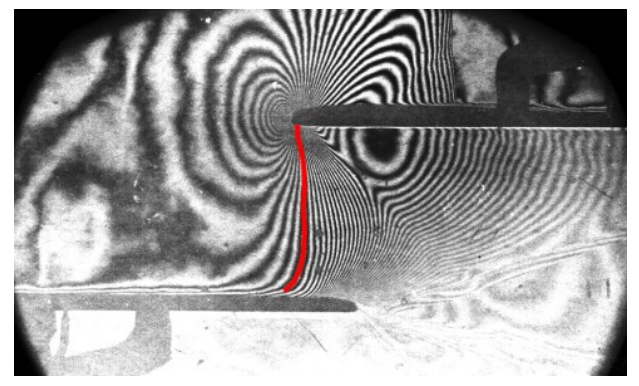

Fig. 13. The sonic line in the tip section of the rotor wheel consisting of blade $1080 \mathrm{~mm}$ long. Interferometric measurement, exit isentropic Mach number $M_{2 i s}=1.83$, inlet Mach number $M_{1}=0.906[15]$.

These reflected compression characteristics are reflected again from the straight surface of the profile and keep the compression character. This way the region of supersonic compression arises between the point of discontinuous curvature of the suction side and the point of impact of the second neutral characteristic (see Figure 12). It is valid in general that the extent of recompression area depends on the shape of the sonic line.

\subsection{Tip section}

The pitch/chord ratio $(s / c)$ reaches larger values in the tip sections, it often exceeds the value $s / c=1$, what means that the inter-blade channel vanishes. The stagger angle $\gamma$ reaches high values and often even exceeds the value $\gamma=80^{\circ}$. The inlet velocity in the tip region significantly grows. Not only is the velocity approaching the sonic value, it often exceeds it. If the inlet Mach number $M_{1}$ is lower than the value $M_{1}=1$, the sonic line links the adjacent profiles and is shifted in the inlet part of the inter-blade channel. It is rising next to the leading edge on the suction side and ends on the pressure side just upstream the trailing edge (see examples on the Figures 11 and 13). The shape of sonic line in the case of cascades consisting of flat profiles (similar to the profile in the Figure 13) depends on stagger angle, pitch/chord ratio and inlet flow angle. The selected results of experimental research are shown in the Figure 14. 
The sets of profile cascades intended for even longer rotor blades usually work with supersonic inlet flow field. The process of the further sonic line formation is well known and has been described by Dvorak [6].
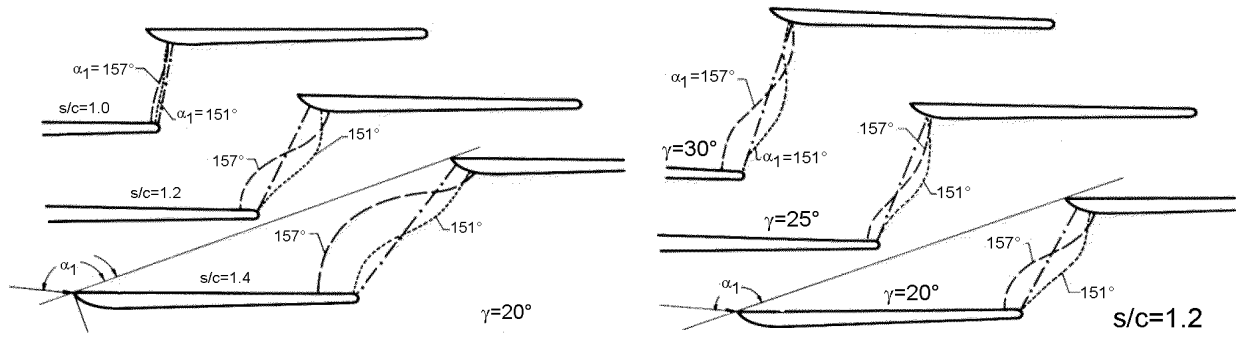

Fig. 14. The dependence of the sonic line shape in the tip section cascade on different values of inlet flow angle $\alpha_{l}$ and different geometric parameters of the cascade (stagger angle $\gamma$, pitch/chord ratio $s / c)$, subsonic inlet Mach number [7].

The topologic description of sonic line in the case of supersonic inlet is different for unstarted and started inlet supersonic flow.

The unstarted inlet supersonic flow field is characterized usually by relatively distant position of the inlet shock wave from the leading edge. The inner branch of the shock wave is ordinarily approximately normal to the pressure side of the adjacent profile in the beginning of unstarted supersonic flow regime. The sonic line is closed in a typical form around the leading edge. An example is shown in the interferometric picture in the Figure 15.

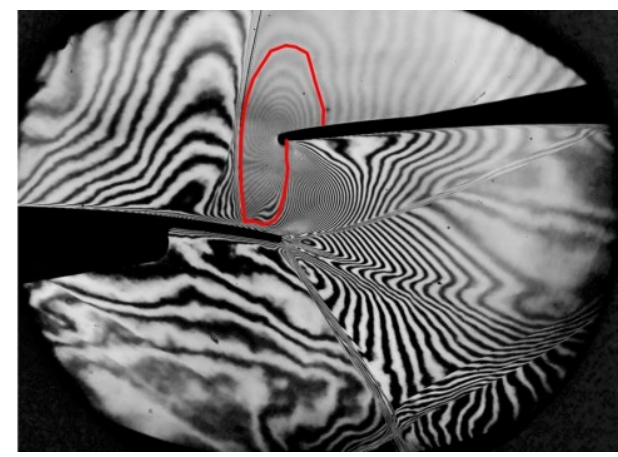

Fig. 15. The sonic line in a tip section with supersonic inlet. Unstarted supersonic inlet flow, exit isentropic Mach number $M_{2 i s}=1.815$, inlet Mach number $M_{1}=1.089$ [16].

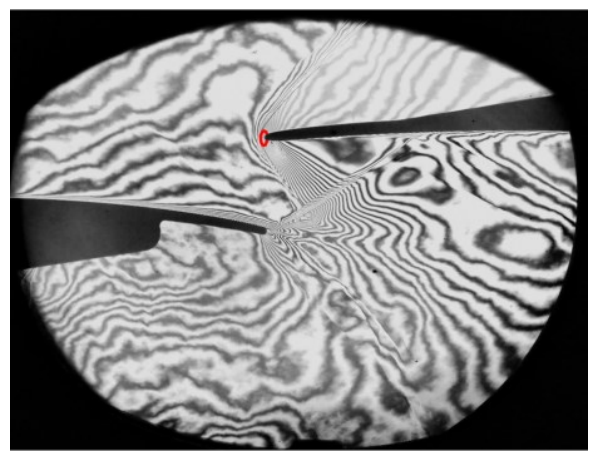

Fig. 16. The sonic line in a tip section with supersonic inlet. Started supersonic inlet flow, exit isentropic Mach number $M_{2 i s}=1.800$, inlet Mach number $M_{1}=1.665$ [16].

The transition from unstarted to started flow is unsteady process in which even the bistability effects appear [6]. The unsteady behaviour of the flow field naturally has an impact on the sonic line. When the inlet Mach number is sufficiently high and the backpressure downstream the cascade is fitting low, the started supersonic flow field takes place in the cascade. Then the sonic line surrounds a small subsonic area near the leading edge (see Figure 16). This subsonic area bordered with sonic line is stable structure and does not disappear even if the supersonic inlet Mach number noticeably increases. The reason lies in the thick leading edge, which is typical for the tip section of rotor long blades intended for last stages of steam turbines. 


\section{Conclusions}

The sonic surface is important aerodynamic threshold in the complex flow field, which occurs in the inter-blade channel of modern last stage rotor wheels blading consisting of long blades. The position and shape of sonic line depends not only on the cascade geometry and inlet and outlet boundary condition (inlet Mach number, inlet flow angle, backpressure) but often also on the effective geometric shape of the cascade profiles. The notion of sonic line as a boundary of constant static pressure is important for proper design of supersonic parts of the blade profiles with line segment in the transonic part of the suction side side of the profile.

The sonic line in the cascade with subsonic inlet Mach number spans across the interblade channel. If there is no overlapping of profiles and inter-blade channel is "missing" in the cascade geometry, the sonic line links the regions of leading and trailing edges of adjacent profiles.

The rapid unsteady transition from unstarted to started inlet supersonic flow field results in small stable subsonic area near the edge surrounded by sonic line (in cascades with thick profiles leading edges).

Acknowledgements of the author belong to the Technology Agency of the Czech Republic, which supported this research under grant No. TH02020057. An institutional support RVO 61388998 is also gratefully acknowledged.

\section{References}

1. M. Luxa, D. Simurda, J. Fort, et al., Aerodynamic Investigation of the the Tip-Section for Titanium Blade 54"'. Proceedings of the 11th European Conference on Turbomachinery, Fluid Dynamics and Thermodynamics (Madrid, 2015)

2. P. Safarik, Application of the Method of Characteristics in Calculation of the Flow in Blade Cascades (in Czech). Semináře o problémech parních turbín 3, ČVTS (Prague, 1976)

3. P. Safarik, Flow in Supersonic Exit Part of the Blade Cascade (in Czech). Research report IT CAS No.: Z-409/73 (Prague, 1973)

4. A.H. Shapiro, The Dynamics and Thermodynamics of Compressible Fluid Flow (1953)

5. P.A. Thompson, Transonic Flow in Curved Channels. Transactions of ASME, J. of Basic Engineering 12 (1967)

6. R. Dvorak, Transonic Flow (in Czech), (Prague, 1987)

7. C. Sieverding, Transonic Turbine Bladings. Privat communications

8. D. Simurda, M. Luxa, P. Safarik, et al., Aerodynamic Data for Two Variants of Root Turbine Blade Sections for a 54" Turbine Rotor Blade. Proceedings of the ASME Turbo Expo, Vol. 2C, No: V02CT38A007 (Düsseldorf, 2014)

9. M. Luxa, D. Šimurda, Optical Measurement on TR-P-3 Cascade (in Czech). Research report IT CAS No.: Z -1492/13 (Prague, 2013)

10. J. Hala, M. Luxa, D. Simurda, et al., Optimization of Root Section for Ultra-Long Turbine Rotor Blade. J. of Thermal Science, Vol. 27, No. 2 (2018)

11. M. Luxa, J. Hala, D. Simurda, Optical Measurement on TR-P-4 Cascade. Research report IT CAS No.: Z-1558/16 (Prague, 2016)

12. T. Radnic, J. Furst, M. Luxa, et al., Aerodynamic Effects of Tie-Boss in Extremely Long Turbine Blades. J Eng Gas Turb Power. GTP-17-1218 (2018) 
13. M. Luxa, D. Simurda, P. Safarik, et al., High-Speed Aerodynamics Investigation of the Midsection of $48^{\prime \prime}$ Rotor Blade for the Last Stage of Steam Turbine. Proceedings of the 10th European Conference on Turbomachinery - Fluid Dynamics and Thermodynamics, pp. 360-369, (Lappeenranta, 2013)

14. R. Dvorak, The Aerodyanmic Properties of Profile Cascades (TR-B-1,2,3) Representing Tip Sections of Turbine Rotor Blading (in Czech). Research report IT CAS No.: Z-423/73 (Prague, 1973)

15. R. Dvorak, P. Safarik, Aerodynamic Research of the Tip Section of Last Stage Rotor Blades for Steam Turbines od Large Output. In: ASME Paper No.: C185/79 (ASME, 1979)

16. M. Luxa, D. Simurda, Optical Measurement on TR-U-6 Cascade. Research report IT CAS No.: Z-1535/15 (Prague, 2015) 